\title{
Odia Text Summarization using Stemmer
}

\author{
R. C. Balabantaray \\ CLIA Lab \\ IIIT, Bhubaneswar \\ Odisha, India
}

\author{
B. Sahoo \\ CLIA Lab \\ IIIT, Bhubaneswar \\ Odisha, India
}

\author{
D. K. Sahoo \\ CLIA Lab \\ IIIT, Bhubaneswar \\ Odisha, India
}

\author{
M. Swain \\ CLIA Lab \\ IIIT, Bhubaneswar \\ Odisha, India
}

\begin{abstract}
Lot of work has already been done for automatic text summarization. In this paper we have given a novel statistical approach to summarize the given Odia text. In our approach extraction of relevant sentences is done which can give the actual concept of the input document in a concise form. We rank each sentence in the document by assigning a weight value to each word of the sentence. The sentences are extracted as per their rank, which will lead to a good summary of the given text.
\end{abstract}

\section{Keywords}

Automatic text summarization, sentence extraction, term weight, Stemmer.

\section{INTRODUCTION}

In the world of information, the increasing availability of online information has necessitated intensive research in the area of automatic text summarization within the field of Natural Language Processing (NLP). In this first growing information age, Text summarization has become an important and timely tool for assisting and interpreting text information.

With the huge availability of text document in the internet, it gives more information than is needed. It is very difficult for human beings to manually summarize large documents of text. So searching for relevant documents through an overwhelming number of documents available in the web is a very difficult task. In order to solve the above two problems, the automatic text summarization is very much necessary.

According to the 8th schedule of Indian constitution there are 22 major languages of India which are recognized by Indian constitution. Odia (Officially changed from Oriya to Odia from November 2011) is one of them which is mainly spoken by the people live in the state Odisha (Officially changed from Orissa to Odisha from November 2011). It belongs to the Indo-Aryan language of the Indo-European language family. It is the official language of Odisha and the second official language of Jharkhand. Though it is an official Indian language, and it is spoken by 31 million people $(83.33 \%$ of total population of Odisha) in Odisha, and some other neighbor states like West Bengal, Jharkhand, Chhattisgarh and Andhra Pradesh. There are also significant number of Odia speaking people in main cities of India i.e. Kolkata, Mumbai, Delhi, Chennai, Bangalore, Hyderabad, Pune, Pondicherry, Gurgaon etc. Odia is not only spoken in India. Odia people are also in some of the prominent countries of the World e.g. USA, UK, Canada, UAE, Srilanka, Singapore, Malaysia, Burma, Indonesia. There are 45 million Oriya speaking people living in globally. So we are motivated towards Odia language and working for this language.

Before going to the Text summarization, first we, have to know that what is a summary. A summary [2] can be employed in an indicative way as a pointer to some parts of the original document, or in an informative way to cover all relevant information of the text. In both cases the most important advantage of using a summary is its reduced reading time.

Text Summarization methods can be classified into extractive and abstractive summarization. An extractive summarization method consists of selecting important sentences, paragraphs etc. from the original document and concatenating them into shorter form. The importance of sentences is decided based on statistical and linguistic features of sentences. An Abstractive summarization [9][10] attempts to develop an understanding of the main concepts in a document and then express those concepts in clear natural language. It uses linguistic methods to examine and interpret the text and then to find the new concepts and expressions to best describe it by generating a new shorter text that conveys the most important information from the original text document. In this paper we focus on novel techniques which are based on extractive text summarization methods.

In this paper, section-2 consists of related works, section-3 consists of our recent work, section- 4 consists of methodology and the algorithm, section-5 consists of result and discussion and finally section- 6 consists of conclusion and future work.

\section{RELATED WORKS}

In this section, we primarily aim to investigate the empirical methods that have been used to build summarization systems. Basically, summarization task is done in two different methods, i.e. extractive and abstractive.

Earliest instances of research on summarizing scientific documents proposed paradigms for extracting salient sentences from text using features like word and phrase frequency (Luhn, 1958),[3] position in the text (Baxendale, 1958) [4]and key phrases (Edmundson, 1969)[5].

Most early work on single-document summarization focused on technical documents. (Luhn, 1958),[3] in his work proposed that the frequency of a particular word in an article provides an useful measure of its significance. There are several key ideas put forward in this paper that have assumed importance in later work on summarization. As a first step, words were stemmed to their root forms, and stop words were deleted. Luhn then compiled a list of content words sorted by decreasing frequency, the index providing a significance measure of the word. On a sentence level, a significance factor was derived that rejects the number of occurrences of 
significant words within a sentence, and the linear distance between them due to the intervention of non-significant words. All sentences are ranked in order of their significance factor, and the top ranking sentences are finally selected to form the auto-abstract.

Related work (Baxendale, 1958)[4], also done at IBM and published in the same journal, provides early insight on a particular feature helpful in finding salient parts of documents: the sentence position. Towards this goal, the author examined 200 paragraphs to find that in $85 \%$ of the paragraphs the topic sentence came as the first one and in $7 \%$ of the time it was the last sentence. Thus, a naive but fairly accurate way to select a topic sentence would be to choose one of these two. This positional feature has since been used in many complex machine learning based systems.

Edmundson (1969)[5] describes a system that produces document extracts. His primary contribution was the development of a typical structure for an extractive summarization experiment. At first, the author developed a protocol for creating manual extracts, that was applied in a set of 400 technical documents. The two features of word frequency and positional importance were incorporated from the previous two works. Two other features were used: the presence of cue words (presence of words like significant, or hardly), and the skeleton of the document (whether the sentence is a title or heading). Weights were attached to each of these features manually to score each sentence. During evaluation, it was found that about $44 \%$ of the auto-extracts matched the manual extracts.

The Trainable Document Summarizer [7] in 1995 performs sentence extracting task, based on a number of weighting heuristics. Following features were used and evaluated:

1. Sentence Length Cut-O Feature: sentences containing less than a pre-specified number of words are not included in the abstract

2. Fixed-Phrase Feature: sentences containing certain cue words and phrases are included

3. Paragraph Feature: this is basically equivalent to Location Method feature in [8]

4. Thematic Word Feature: the most frequent words are defined as thematic words. Sentence scores are functions of the thematic words' frequencies

5. Uppercase Word Feature: upper-case words (with certain obvious exceptions) are treated as thematic words, as well.

The ANES text extraction system [8] in 1995 is a system that performs automatic, domain-independent condensation of news data. The process of summary generation has four major constituents:

1. Corpus analysis: this is mainly a calculation of the tf*idf weights for all terms

2. Statistical selection of signature words: terms with a high tf*idf-weight plus headline-words

3. Sentence weighting: summing over all signature word weights, modifying the weights by some other factors, such as relative location

4. Sentence selection: Selecting high scored sentences.

\section{OUR RECENT WORK}

In this paper we use the extractive method to get the summary of the input document. In order to extract the summary, we use the following features:
1. Content (Key) words: After removing the stop words the remaining words are treated as key words. We have taken the total number of key word during assigning the weight to each term.

2. Frequent key word occurrence in the text: The frequency of the key word which are frequently occurred in the document.

3. Sentence location feature: Usually first sentence of first paragraph of a text document are more important and are having greater chances to be included in summary. So in our case we have made the inclusion of first sentence of the first paragraph of the document is mandatory.

\section{METHODOLOGY}

Our summarizer takes input in text format i.e. .txt . Firstly it tokenizes the text in order to find the individual tokens or terms. Then we are filtering the text by removing the stop words. After removing the stop words we stem each word by using an Odia stemmer. After that a weight value is assigned to each individual term. The weight is calculated as follows: The weight,

$w t=\frac{\text { frequency of the term }}{\text { Total no. of terms in the document }}$

After assigning the weight to each term, the next job is to ranking the individual sentence according to their weight value. The weight of the sentence can be calculated by adding the weight of all the terms in the sentence and dividing it by total number of terms in that sentence, i.e.

$$
w t_{s}=\sum_{i=1}^{\mathrm{n}}\left(w t_{i}\right) / \mathrm{n}
$$

Where $w t_{s}=$ weight of the sentence.

$w t_{1}, w t_{2}, w t_{3}, \ldots \ldots \ldots t_{n}$ are the weights of individual terms in that sentence.

$\mathrm{n}=$ total number of terms in that sentence.

A word has different form. Whenever we assign the weight value we consider the different forms of a word as individual terms. So the average weight of the sentences becomes less. We are using an Odia stemmer which gives the stem or rootword of each individual term, so that the average weight of the sentence can be more. Our stemmer uses suffix and prefix removal method to give the stemmed word.

Finally, our summarizer extracts the higher rank sentences including the first sentence of the first paragraph of the document. The number of sentences extracted is based on the user requirement i.e. the percentages of summary the user give as input. This percentage is calculated by dividing the percentage given by the user by total number of ranked sentences, and then taking the ceiling of that result.

\subsection{Algorithm}

Input: A text in .txt format.

Output: A relevant summarized text which is shorter than the original text keeping the theme or concept constant. 
1. Read a Odia text in .txt format and split it into individual tokens.

2. Remove the stop words to filter the text.

3. Stem individual words by using the Odia Stemmer.

4. Assign a weight value to each individual terms. The weight is calculated as:

$$
W t=\frac{\text { frequency of the term }}{\text { Total no.of terms in the document }}
$$

5. Rank the individual sentences according to their weight value as :

$$
w t_{s}=\sum_{i=1}^{\mathrm{n}}\left(w t_{i}\right) / \mathrm{n}
$$

Where $W t_{s}=$ weight of the sentence. $w t_{1}, w t_{2}, w t_{3}, \ldots \ldots \ldots t_{n}$ are the

weights of individual term in that sentence.

$\mathrm{n}=$ total number of terms in that sentence.

6. Finally, extract the higher ranked sentences including the first sentence of the first paragraph of the input text in order to find the required summary. The number of sentences extracted is based on the user requirement i.e. the percentages of summary given as input.

\section{RESULT AND DISCUSSION}

We have tested our system with 20 documents. Here each document contains around 30 sentences. For auto summarization we have fixed the percentage of summary as $50 \%$, i.e. it will reduce the summary to half of the original document. The relevancy of the summary is calculated with respect to (w.r.t.) human judgment for the system. The Screen shot of our system is given in figure- 1 and the details of the result are given in the table- 1 which are given below. The relevancy of our system with respect to human judgement is plotted in figure-2.

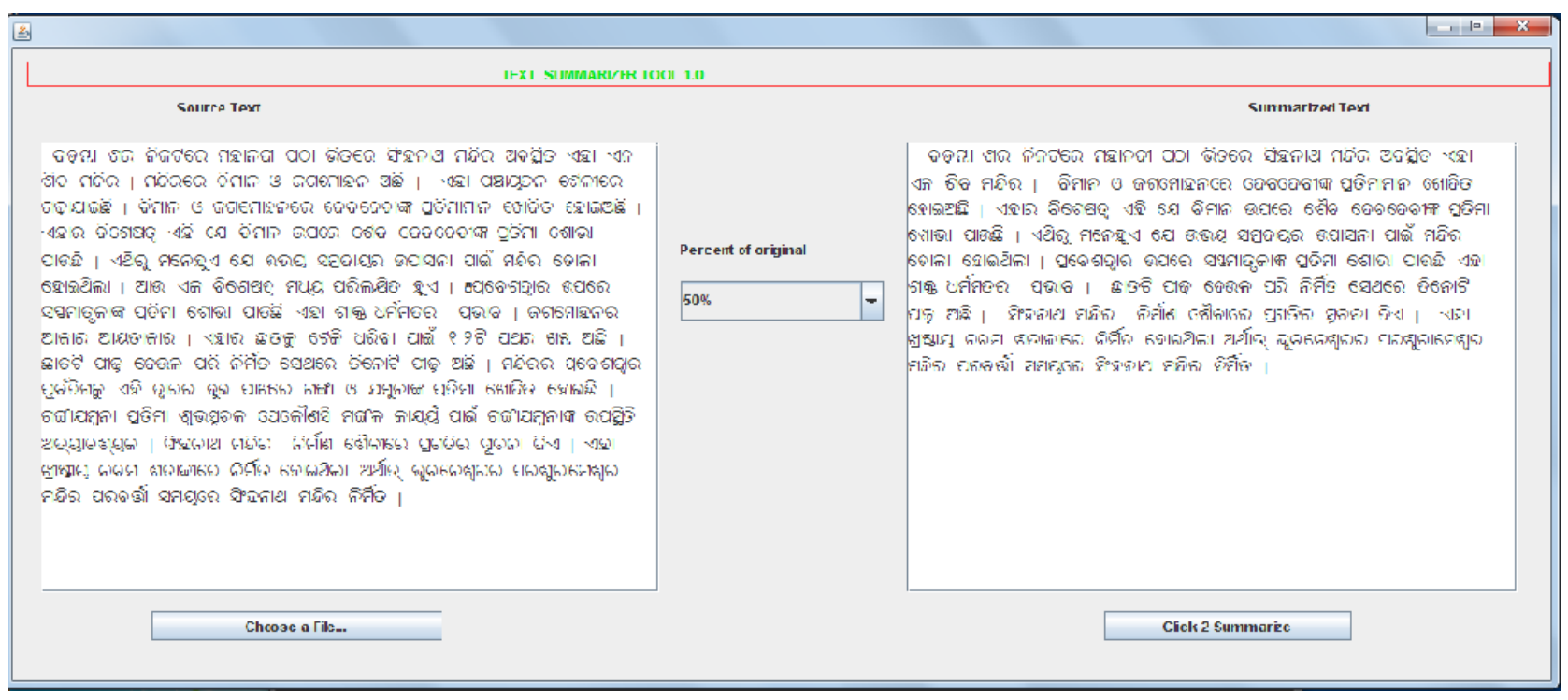

Fig 1: Screen Short of our System

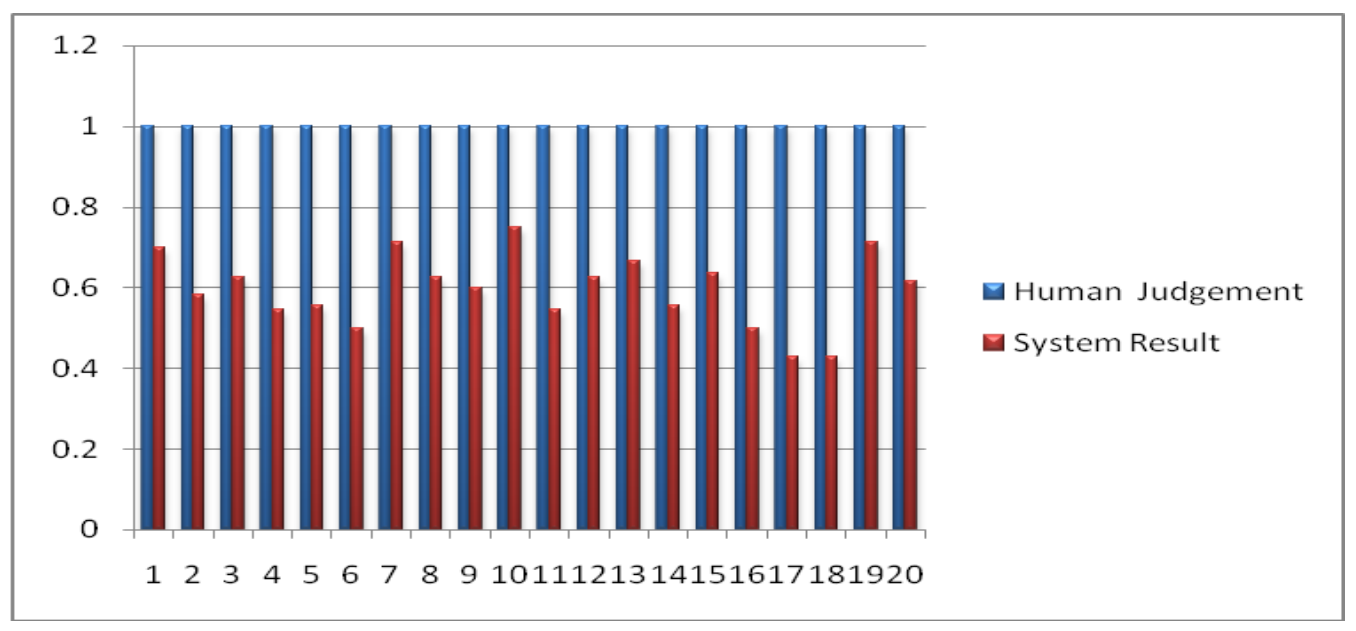

Fig 2: Relevancy of our System w.r.t. Human Judgement 
Table 1. Result Details

\begin{tabular}{|c|c|c|c|}
\hline $\begin{array}{l}\text { Docume } \\
\text { nt } \\
\text { Name(N } \\
\text { o. of } \\
\text { Sentenc } \\
\text { es } \\
\text { present } \\
\text { in the } \\
\text { docume } \\
\text { nt) }\end{array}$ & $\begin{array}{l}\text { No. of } \\
\text { Sentences } \\
\text { extracted by } \\
\text { our System } \\
\text { (Sentence No.) }\end{array}$ & $\begin{array}{l}\text { No. of } \\
\text { Sentences } \\
\text { extracted by } \\
\text { Human Analysis } \\
\text { (Sentence No.) }\end{array}$ & $\begin{array}{l}\text { Relevance } \\
\text { for Our } \\
\text { System } \\
\text { w.r.t. } \\
\text { Human } \\
\text { Analysis }\end{array}$ \\
\hline $\begin{array}{l}\text { Text1.txt } \\
\text { (20) }\end{array}$ & $\begin{array}{l}10(1,6,7,9,11,1 \\
2,15,17,18,19)\end{array}$ & $\begin{array}{l}10(1,3,7,8,10,11 \\
12,17,18,19)\end{array}$ & 0.700 \\
\hline $\begin{array}{l}\text { Text2.txt } \\
(24)\end{array}$ & $\begin{array}{l}12(1,8,9,12,13 \\
14,15,16,17,19 \\
20,23)\end{array}$ & $\begin{array}{l}12(1,2,3,4,6,11,1 \\
4,15,16,17,20,23 \\
)\end{array}$ & 0.583 \\
\hline $\begin{array}{l}\text { Text3.txt } \\
\text { (16) }\end{array}$ & $\begin{array}{l}8(1,3,4,6,10,12 \\
13,14)\end{array}$ & $\begin{array}{l}8(1,2,3,6,9,10,14 \\
)\end{array}$ & 0.625 \\
\hline $\begin{array}{l}\text { Text4.txt } \\
(22)\end{array}$ & $\begin{array}{l}11(1,3,4,7,8,9,1 \\
2,14,15,18,21)\end{array}$ & $\begin{array}{l}11(1,2,4,7,9,10,1 \\
1,12,13,18,19)\end{array}$ & 0.545 \\
\hline $\begin{array}{l}\text { Text5.txt } \\
\text { (18) }\end{array}$ & $\begin{array}{l}9(1,2,3,4,6,8,11 \\
, 14,17)\end{array}$ & $\begin{array}{l}9(1,2,5,8,10,13,1 \\
4,16,17)\end{array}$ & 0.555 \\
\hline $\begin{array}{l}\text { Text6.txt } \\
(28)\end{array}$ & $\begin{array}{l}14(1,2,5,6,7,8,9 \\
, 11,14,20,21,23 \\
, 25,26)\end{array}$ & $\begin{array}{l}14(1,3,4,5,7,10,1 \\
3,14,15,18,22,24, \\
25,26)\end{array}$ & 0.500 \\
\hline $\begin{array}{l}\text { Text7.txt } \\
\text { (14) }\end{array}$ & $\begin{array}{l}7(1,3,6,8,9,11,1 \\
3)\end{array}$ & $\begin{array}{l}7(1,3,4,7,8,11,13 \\
)\end{array}$ & 0.714 \\
\hline $\begin{array}{l}\text { Text8.txt } \\
(16)\end{array}$ & $\begin{array}{l}8(1,2,3,4,5,12,1 \\
3,14)\end{array}$ & $\begin{array}{l}8(1,2,3,5,7,8,9,1 \\
3)\end{array}$ & 0.625 \\
\hline $\begin{array}{l}\text { Text9.txt } \\
(20)\end{array}$ & $\begin{array}{l}10(1,3,4,5,6,9,1 \\
1,1,2,16,18)\end{array}$ & $\begin{array}{l}10(1,2,5,6,9,10,1 \\
2,15,16,18)\end{array}$ & 0.600 \\
\hline $\begin{array}{l}\text { Text10.t } \\
x t(16)\end{array}$ & $\begin{array}{l}8(1,2,4,5,6,8,11 \\
12)\end{array}$ & $\begin{array}{l}8(1,2,3,5,6,9,11 \\
12)\end{array}$ & 0.750 \\
\hline $\begin{array}{l}\text { Text11.t } \\
x t(22)\end{array}$ & $\begin{array}{l}11(1,2,4,5,7,8,1 \\
0,13,15,16,21)\end{array}$ & $\begin{array}{l}11(1,3,6,7,11,12, \\
13,15,16,20,21)\end{array}$ & 0.545 \\
\hline $\begin{array}{l}\text { Text12.t } \\
x t(16)\end{array}$ & $\begin{array}{l}8(1,2,4,5,8,12,1 \\
, 3,15)\end{array}$ & $\begin{array}{l}8(1,2,4,5,6,7,10, \\
12)\end{array}$ & 0.625 \\
\hline $\begin{array}{l}\text { Text13.t } \\
x t(18)\end{array}$ & $\begin{array}{l}9(1,3,4,6,8,13,1 \\
4,16,19,20)\end{array}$ & $\begin{array}{l}9(1,3,5,6,8,9,14, \\
17,20)\end{array}$ & 0.666 \\
\hline $\begin{array}{l}\text { Text14.t } \\
x t(18)\end{array}$ & $\begin{array}{l}9(1,2,3,5,6,7,10 \\
13,15)\end{array}$ & $\begin{array}{l}9(1,2,4,5,8,10,12 \\
, 14,15)\end{array}$ & 0.555 \\
\hline $\begin{array}{l}\text { Text15.t } \\
x t(22)\end{array}$ & $\begin{array}{l}11(1,2,3,4,5,13, \\
15,16,17,9,20)\end{array}$ & $\begin{array}{l}11(1,2,3,4,6.10,1 \\
1,12,16,17,20)\end{array}$ & 0.636 \\
\hline $\begin{array}{l}\text { Text16.t } \\
x t(24)\end{array}$ & $\begin{array}{l}12(1,2,3,4,6,9,1 \\
2,13,15,16,17,2 \\
2)\end{array}$ & $\begin{array}{l}12(1,2,3,5,7,9,10 \\
14,15,18,19,22)\end{array}$ & 0.500 \\
\hline $\begin{array}{l}\text { Text17.t } \\
x t(14)\end{array}$ & $\begin{array}{l}7(1,3,4,5,11,13, \\
14)\end{array}$ & $7(1,2,4,5,7,9,10)$ & 0.428 \\
\hline $\begin{array}{l}\text { Text18.t } \\
x t(14)\end{array}$ & $\begin{array}{l}7(1,3,4,9,11,12, \\
13)\end{array}$ & $7(1,2,3,6,7,9,10)$ & 0.428 \\
\hline $\begin{array}{l}\text { Text19.t } \\
x t(14)\end{array}$ & $\begin{array}{l}7(1,4,5,6,8,13,1 \\
4)\end{array}$ & $\begin{array}{l}7(1,3,4,5,8,11,14 \\
)\end{array}$ & 0.714 \\
\hline $\begin{array}{l}\text { Text20.t } \\
x t(26)\end{array}$ & $\begin{array}{l}13(1,4,5,6,7,8,1 \\
5,16,18,19,20,2 \\
2,23)\end{array}$ & $\begin{array}{l}13(1,2,4,5,7,8,11 \\
12,14,15,19,23,2 \\
4)\end{array}$ & 0.615 \\
\hline
\end{tabular}

\section{CONCLUSION AND FURURE WORK}

In this paper we use the extraction method for Odia text summarization. We have extracted the sentences according to their weight by maintaining their proper order. Here the first sentence is made mandatory to extract because it is considered as an important sentence of the given text document.

Since the summarization follows the extraction method, when it extract the important sentences it might happen that one sentence contains a proper noun and at the next sentence contains a pronoun as a reference of the proper noun. In that case, if the summary considers the second sentence without considering the first one, then it does not give its proper meaning. It is a big issue in automatic text summarization. We are working on it to resolve this type of anaphoric problems in text summarization.

\section{ACKNOWLEDGEMENT}

We are much indebted to The Department of Information Technology (DIT), Ministry of Communication and Information Technology(MCIT), Govt. of India for this research work.

\section{REFERENCE}

[1] Farshad Kyoomarsi, Hamid Khosravi, Esfandiar Eslami and Pooya Khosravyan Dehkordy, "Optimizing Text Summarization Based on Fuzzy Logic", In proceedings of Seventh IEEE/ACIS International Conference.

[2] Weiguo Fan, Linda Wallace, Stephanie Rich, and Zhongju Zhang, "Tapping into the Power of Text Mining”, Journal of ACM, Blacksburg, 2005.

[3] Luhn, H. P. (1958). The automatic creation of literature abstracts. IBM Journal of Research Development, 2(2):159-165.

[4] Baxendale, P. (1958). Machine-made index for technical literature - an experiment. IBM Journal of Research Development, 2(4):354-361.

[5] Edmundson, H. P. (1969). New methods in automatic extracting. Journal of the ACM, 16(2):264-285.

[6] H. P. Edmundson.," New methods in automatic extracting", Journal of the ACM, 16(2):264-285, April 1969.

[7] J. Kupiec, J. Pedersen, and F. Chen, "A trainable document summarizer", In Proceedings of the 18th ACMSIGIR Conference, pages 68-73, 1995.

[8] Ronald Brandow, Karl Mitze, and Lisa F. Rau. "Automatic condensation of electronic publications by sentence selection. Information Processing and Management", 31(5):675-685,1995.

[9] Vishal Gupta, G.S. Lehal, "A Survey of Text Mining Techniques and Applications", Journal of Emerging Technologies in Web Intelligence, VOL. 1, NO. 1, 6076, AUGUST 2009.

[10] G Erkan and Dragomir R. Radev, "LexRank: Graphbased Centrality as Salience in Text Summarization", Journal of Artificial Intelligence Research, Re-search, Vol. 22, pp. 457-479 2004.

[11] Udo Hahn and Martin Romacker, "The SYNDIKATE text Knowledge base generator",Proceedings of the first International conference on Human language technology research, Association for Computational Linguistics, ACM, Morristown, NJ, USA, 2001. 